Revue des patrimoines

$41 \mid 2019$

Patrimoines gastronomiques. Définitions, typologies et enjeux de conservation

\title{
La fabrique du patrimoine gastronomique dans les musées départementaux de l'Ain : une aventure croisée entre botanique, histoire, ethnologie et arts
}

Promoting the gastronomic heritage in the museums of the Ain department, an adventure with botanical, historical, ethnological and artistic ingredients

\section{Delphine Cano}

\section{(2) OpenEdition}

Journals

\section{Édition électronique}

URL : http://journals.openedition.org/insitu/26190

DOI : 10.4000/insitu.26190

ISSN : 1630-7305

\section{Éditeur}

Ministère de la Culture

\section{Référence électronique}

Delphine Cano, «La fabrique du patrimoine gastronomique dans les musées départementaux de l'Ain : une aventure croisée entre botanique, histoire, ethnologie et arts », In Situ [En ligne], 41 | 2019, mis en ligne le 13 décembre 2019, consulté le 10 décembre 2020. URL : http://journals.openedition.org/ insitu/26190; DOI : https://doi.org/10.4000/insitu.26190

Ce document a été généré automatiquement le 10 décembre 2020.

In Situ Revues des patrimoines est mis à disposition selon les termes de la licence Creative Commons Attribution - Pas d'Utilisation Commerciale - Pas de Modification 4.0 International. 


\section{La fabrique du patrimoine gastronomique dans les musées départementaux de l'Ain : une aventure croisée entre botanique, histoire, ethnologie et arts}

Promoting the gastronomic heritage in the museums of the Ain department, an adventure with botanical, historical, ethnological and artistic ingredients

Delphine Cano

\section{L'Ain et la gastronomie : une dialectique à l'œuvre}

\section{L'Ain berceau de la gastronomie}

1 L'Ain est une terre de gastronomie, qui a vu naître Brillat-Savarin, l'un des pères de cette notion, et abrité ou accueilli des grandes figures liées au bien manger, aussi diverses que Charles Fourier, Lucien Tendret ou encore Gertrude Stein. Bresse et Bugey y résonnent communément dans la littérature gastronome et touristique comme des régions particulièrement représentatives du bien manger. «La Bresse et le Bugey représentent pour nous la perfection dans l'ordre gastronomique, par la qualité et la variété de la cuisine, par le genre de tables où on la déguste, par l'atmosphère au milieu de laquelle on s'en réjouit $»^{1}$, écrivaient Curnonsky² (fig. 1) et Marcel Rouff. Citons, parmi de nombreux exemples, une autre formule de Curnonsky : « En Bresse, patrie de notre maître Brillat-Savarin et région gastronomique entre toutes, où la cuisine française atteint la perfection $»^{3}$, mais aussi la sentence du journaliste G. Lenotre au sujet de la cuisine bressane ( il ne faut pas moins de vingt siècles de civilisation pour atteindre à cette perfection! $\left.»^{4}\right)$, ou encore la présentation du Guide bleu en 1961, à 
propos de la Bresse et du Bugey: "Cette région, qui se réclame sans déchoir des maîtres Brillat-Savarin et Lucien Tendret, est le véritable paradis des gastronomes. ${ }^{5}$ »

Figure 1

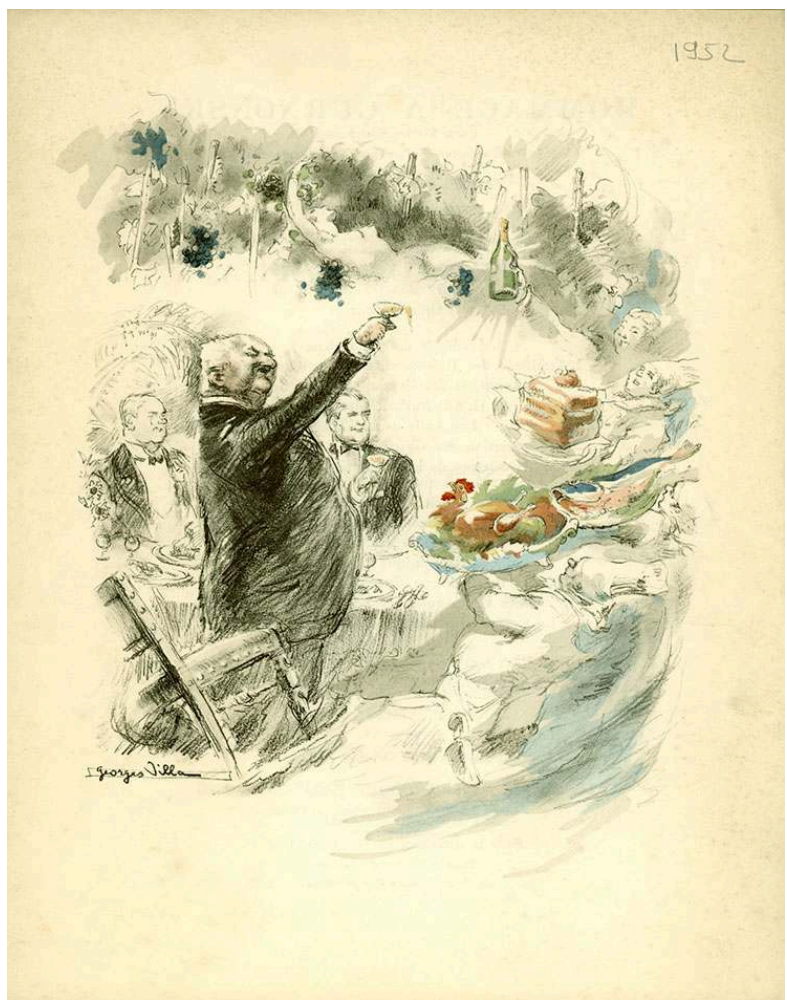

Curnonsky lors d'un toast le 28 octobre 1952. Coll. départementale des Musées de l'Ain, $n^{\circ}$ inv. 2005.17.01

Phot. Jorge Alves. (c) Conseil départemental de l'Ain, Direction des musées départementaux de l'Ain.

2 Le département de l'Ain, au travers de la figure tutélaire de Brillat-Savarin (fig. 2), de ses « mères » et de la réputation de ses « pays » (Bresse, Bugey notamment), est ainsi un territoire étroitement associé à la notion de "gastronomie" mais aussi à celle de qualité de ses produits et de ses traditions culinaires, conçues comme largement partagées et transmises de génération en génération par sa population. 
Figure 2

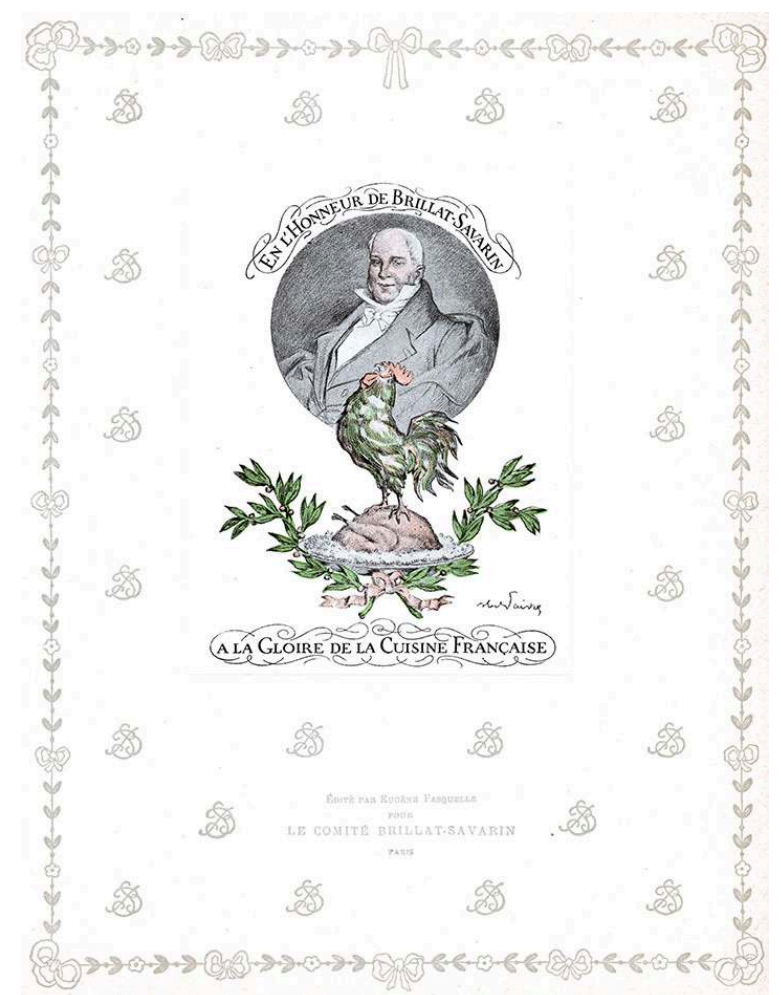

Livret de « la Grande semaine culinaire » de Brillat-Savarin contenant un historique et panégyrique du personnage, et une photographie de Belley, 1921. La quatrième de couverture porte l'inscription suivante en médaillon : «A Brillat-Savarin, les Gourmets de France reconnaissants ». Coll. départementale des Musées de l'Ain, $n^{\circ}$ inv. 99.87.06.

Phot. Jorge Alves. (c) Conseil départemental de l'Ain, direction des Musées départementaux de l'Ain.

3 Nous nous situons ici dans la tradition conceptuelle de la définition large de la gastronomie selon Jean Anthelme Brillat-Savarin, considérée comme

« la connaissance raisonnée de tout ce qui a rapport à l'homme en tant qu'il se nourrit. Son but est de veiller à la conservation des hommes, au moyen de la meilleure nourriture possible. Elle y parvient en dirigeant, par des principes certains, tous ceux qui recherchent, fournissent ou préparent les choses qui peuvent se convertir en aliments. Ainsi, c'est elle, à vrai dire, qui fait mouvoir les cultivateurs, les vignerons, les pêcheurs, les chasseurs et la nombreuse famille des cuisiniers [...]. La gastronomie tient : à l'histoire naturelle [...], à la physique [...] à la chimie [...] à la cuisine [...] au commerce [...], enfin, à l'économie politique [...] ${ }^{6}$.

L'amplitude de cette définition tient à la fois à l'ensemble des champs disciplinaires et économiques qu'elle embrasse et à la largeur du spectre social concerné, à une époque où, dans le sillage de la Révolution française, les pratiques de la cuisine aristocratique se sont diffusées dans la société ${ }^{7}$ et où les cuisines régionales commencent à avoir droit de cité.

\section{La gastronomie comme emblème territorial}

Les propriétés géographiques du territoire de l'Ain, espace d'échanges et de circulation entre Lyon, Genève, la vallée du Rhône et la Bourgogne, sont propices au développement des activités liées à l'alimentation. Terre d'approvisionnement des villes qui l'entourent, l'Ain est également le terrain de jeu de leurs classes aisées, 
comme l'illustre la tradition de la pêche et de la chasse, pratiquées par les propriétaires lyonnais en Dombes (fig. 3). La proximité de la Suisse a permis aussi aux restaurants du Bugey d'être fréquentés par une clientèle internationale et de qualité : ainsi la mère Prusse $^{8}$ à Ameyzieu, au tournant des $\mathrm{xIX}^{\mathrm{e}}$ et $\mathrm{xx}^{\mathrm{e}}$ siècles, accueillit-elle de nombreux Genevois'. De même, dans l'entre-deux-guerres, l'hôtel-restaurant Pernollet, à Belley, gratifié de trois étoiles par le guide Michelin en 1933, a profité de la clientèle drainée par la présence de la Société des Nations à Genève ${ }^{10}$.

Figure 3

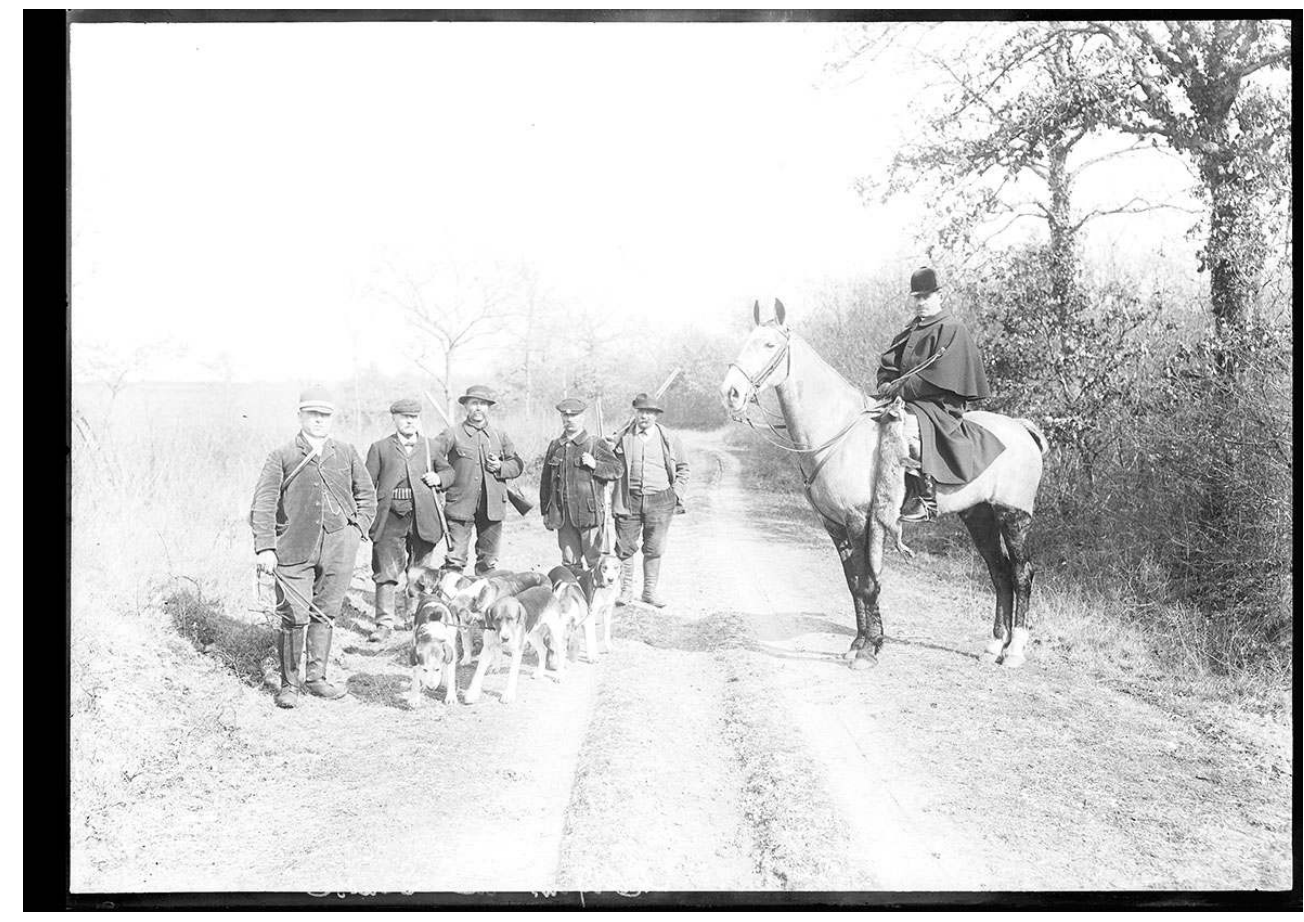

Scène de chasse représentant le marquis de Leusse et son équipage, plaque de verre photographique de Jean-Baptiste Tournassoud, vers 1910. Coll. départementale des Musées de l'Ain,

$n^{\circ}$ inv. 87.13.4654.

Phot. Jorge Alves. (c) Conseil départemental de l'Ain, direction des Musées départementaux de l'Ain.

6 À partir du XIXe siècle, l'émergence et le développement du chemin de fer et des circulations touristiques contribuent également, un peu partout en France, à la réinvention des produits et spécialités régionales, qui participent de la célébration des "petites patries» au sein de la France républicaine ${ }^{11}$ et à la construction d'une représentation collective du territoire national. À leurs côtés, d'autres «icônes " (costumes et mobiliers, spécificités architecturales) participent de la création de marqueurs identitaires régionaux. Tourisme et gastronomie sont ainsi liés par le développement d'une littérature tantôt régionaliste, gastronomique (fig. 4) ou touristique associant de plus en plus intimement l'image d'un territoire avec ses produits et sa cuisine régionale. 
Figure 4

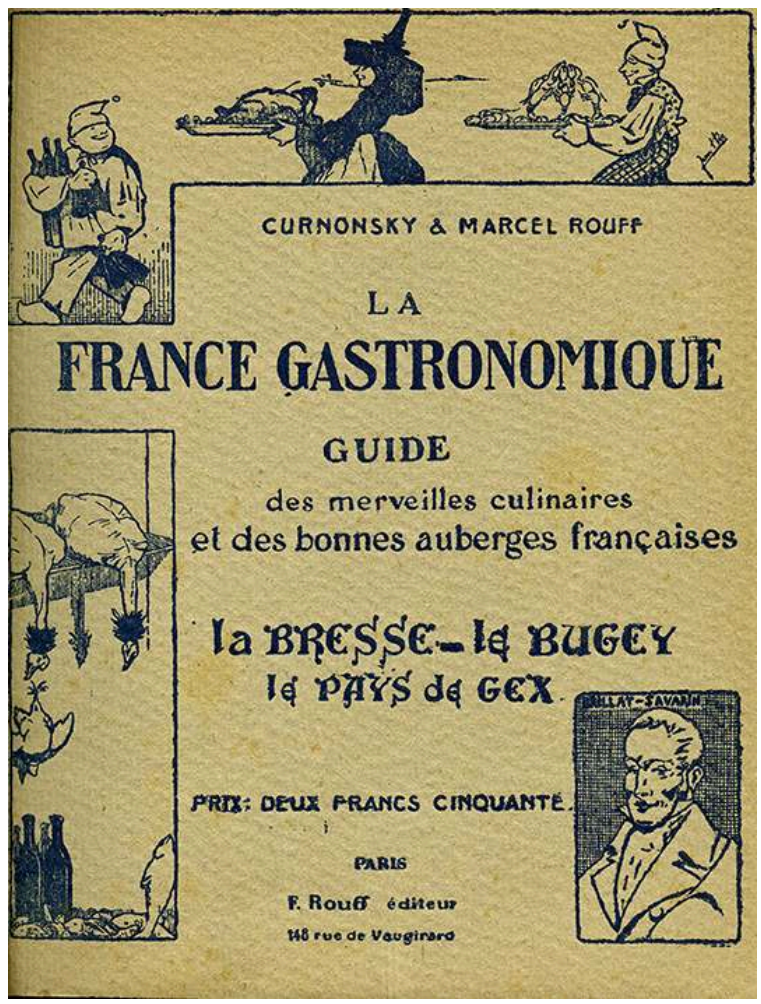

Couverture du tome de La France gastronomique de Curnonsky et Marcel Rouff consacré à la Bresse, au Bugey et au pays de Gex, 1921.

Phot. Jorge Alves. (c) Conseil départemental de l'Ain, direction des Musées départementaux de l'Ain.

Dans l'Ain, la construction de l'identité du territoire passe aussi par sa dimension gastronomique $^{12}$. Certains produits, tout particulièrement la volaille de Bresse, sont revendiqués comme des éléments essentiels du patrimoine local et participent pleinement de l'image iconique "traditionnelle» du territoire, associée au costume bressan ${ }^{13}$ (notamment à son chapeau à cheminée et à ses émaux) et à la ferme à pans de bois, torchis et pisé (fig. 5). D'autres produits au contraire, comme la carpe en Dombes ou les gaudes, aliment quotidien traditionnel associé dans l'imaginaire collectif local à une image de frugalité et de privations, restent plus longtemps dans l'ombre ${ }^{14}$. Ces produits ressurgissent néanmoins aujourd'hui selon une dynamique récente (depuis moins de dix ans), dans le cadre de pratiques d'innovations artisanales et gastronomiques visant à proposer des produits adaptés au goût et aux pratiques du consommateur contemporain, tout en s'appuyant sur la dimension culturelle évocatrice d'enracinement local des produits ${ }^{15}$. 
Figure 5

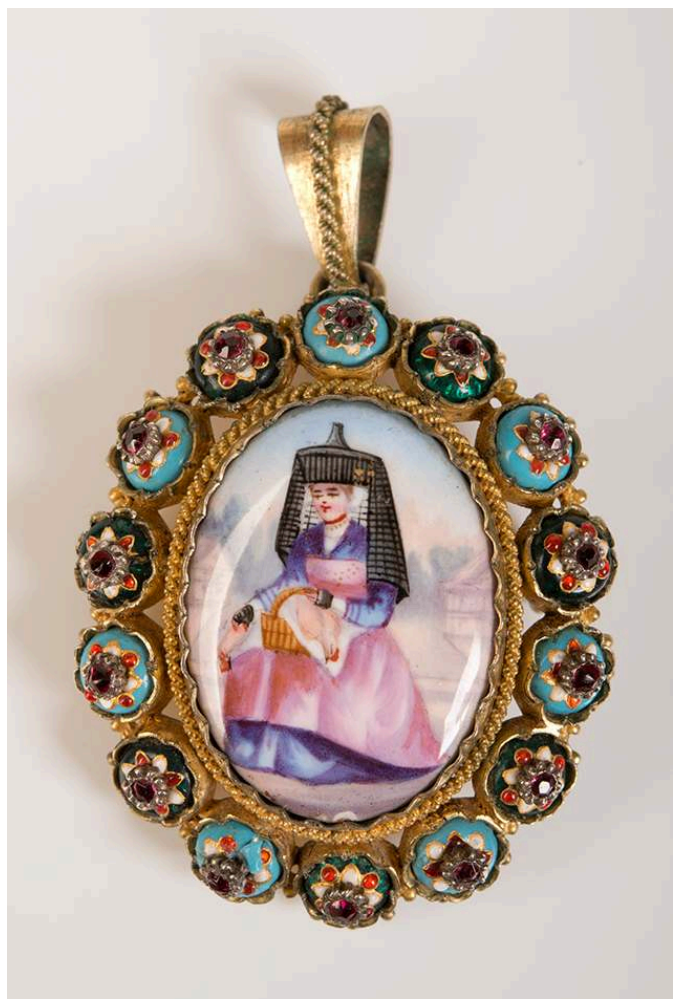

Pendentif-broche en émaux bressans et plaque émaillée centrale représentant une femme en tenue traditionnelle bressane, avec son chapeau à cheminée, son châle et devanti (tablier), tenant sur ses genoux un panier contenant deux volailles; des fermes à pans de bois apparaissent en fond de composition ; vers 1880. Coll. départementale des Musées de l'Ain, nº inv. 2012.22.04.

Phot. Jorge Alves. (c) Conseil départemental de l'Ain, direction des Musées départementaux de l'Ain.

\section{Une promotion économique, touristique et culturelle du patrimoine gastronomique départemental}

8 L'appariement entre gastronomie, tourisme, image du territoire et développement économique s'observe aujourd'hui de façon très actuelle dans la politique menée par le département de l'Ain, visant la croissance du tourisme et du rayonnement territorial, d'une part, et la valorisation de son patrimoine gastronomique, d'autre part, notamment à travers la création récente de la marque "Saveurs de l'Ain». Cette marque vient ainsi apporter une dynamique collective, économique et touristique à la promotion des produits du terroir, soutenue par ailleurs de façon historique par les chefs (fig. 6), les organisations professionnelles et les confréries gourmandes ${ }^{16}$. 
Figure 6

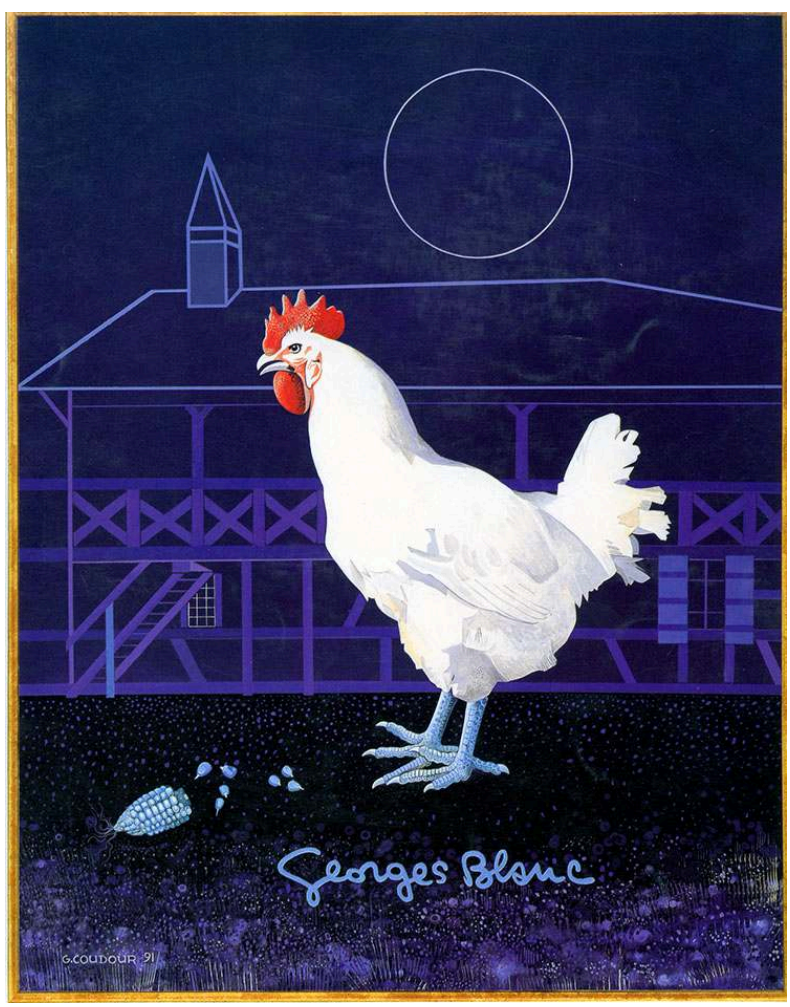

Carte du restaurant Georges Blanc à Vonnas (été 1991) représentant en couverture une illustration de Gilles Coudour avec un poulet de Bresse, produit phare du restaurateur triplement étoilé depuis 1981, devant une stylisation du domaine des Planons. Coll. départementale des Musées de l'Ain, $n^{\circ}$ inv. 91.62.03.

Phot. Jorge Alves. (c) Conseil départemental de l'Ain, direction des Musées départementaux de l'Ain.

C'est également dans ce contexte que s'étudie aujourd'hui le devenir du musée de la Bresse-Domaine des Planons, à Saint-Cyr-sur-Menthon, à proximité du «village gourmand" de Vonnas. Musée ouvert en 1995 dans une ferme bressane classée monument historique ${ }^{17}$ (fig. 7), agrandie en 2005 par la construction d'un bâtiment contemporain, il a été conçu à son origine comme le pôle "Bresse» du musée départemental des Pays de l'Ain, musée « déconcentré » visant à valoriser le patrimoine ethnologique et culturel du département, dans sa diversité de territoires. Ce musée a incorporé très tôt une forte dimension consacrée à l'anthropologie de l'alimentation et a diversifié son approche, faisant dialoguer patrimoine ethnologique avec patrimoines artistiques, caractéristiques alimentaires régionales, arts de la table et «haute gastronomie ». Aujourd'hui, alors que l'engouement pour la gastronomie se traduit par la naissance de quatre Cités de la gastronomie en milieu urbain, le musée fait l'objet d'une réflexion, accompagnée par un conseil scientifique, pour être pleinement repositionné sur la thématique gastronomique et contribuer à la conservation, la connaissance et la valorisation du patrimoine alimentaire du département, à la fois dans ce qu'il a pu apporter à la notion "universelle » de gastronomie mais aussi dans ses spécificités en tant que lieu de consommation et de production. 
Figure 7

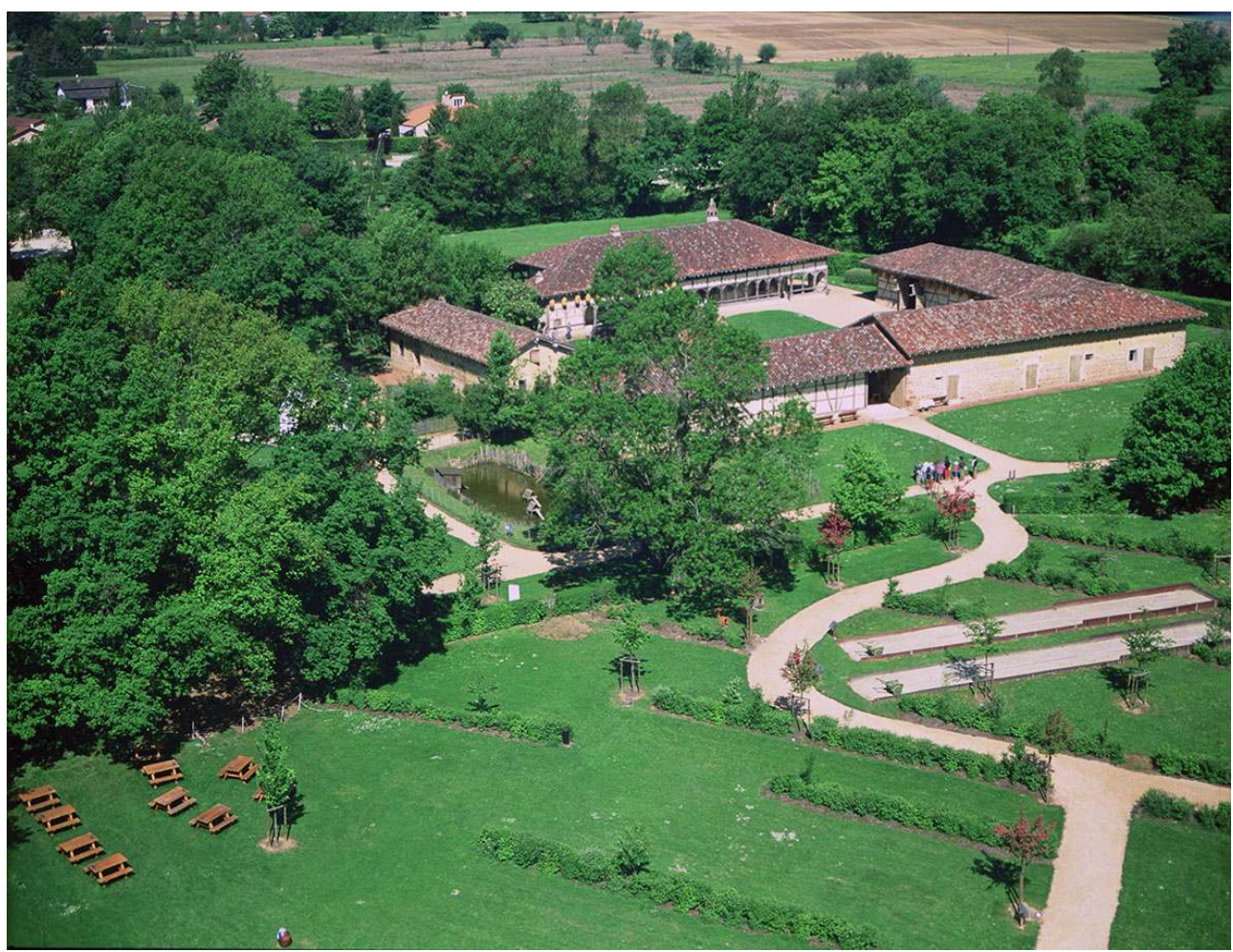

Vue aérienne de la ferme du domaine des Planons, siège du musée de la Bresse depuis 1995, à laquelle s'est adjoint, en 2005, un bâtiment d'architecture contemporaine signé Girodet-Dassonville et Robin.

Phot. O. Zeppeline. (c) Conseil départemental de l'Ain, Direction des musées départementaux de l'Ain.

\section{5 ans de patrimonialisation de la gastronomie au sein des musées départementaux de l'Ain : une analyse rétrospective}

\section{Une approche multi vectorielle issue de l'histoire muséale du Département}

10 La direction des Musées départementaux de l'Ain (anciennement "conservation départementale ») a, dès son origine, en 1984, été confrontée à la question de l'identité gastronomique du territoire et a fait de la gastronomie un élément majeur de sa démarche de conservation et de valorisation du patrimoine départemental. Le patrimoine alimentaire se situant au cœur de la définition des territoires, il est également au centre des collections et des musées d'ethnographie régionale développés dans l'Ain dans les années 1980 et 1990 :

11 - musée du Bugey Valromey (Lochieu), avec la figure de Brillat-Savarin, la vie quotidienne et économique des polyculteurs de moyenne montagne (élevage, vigne, apiculture... $)^{18}$ (fig. 8), la grande cuisine de la maison Renaissance abritant le musée ; 


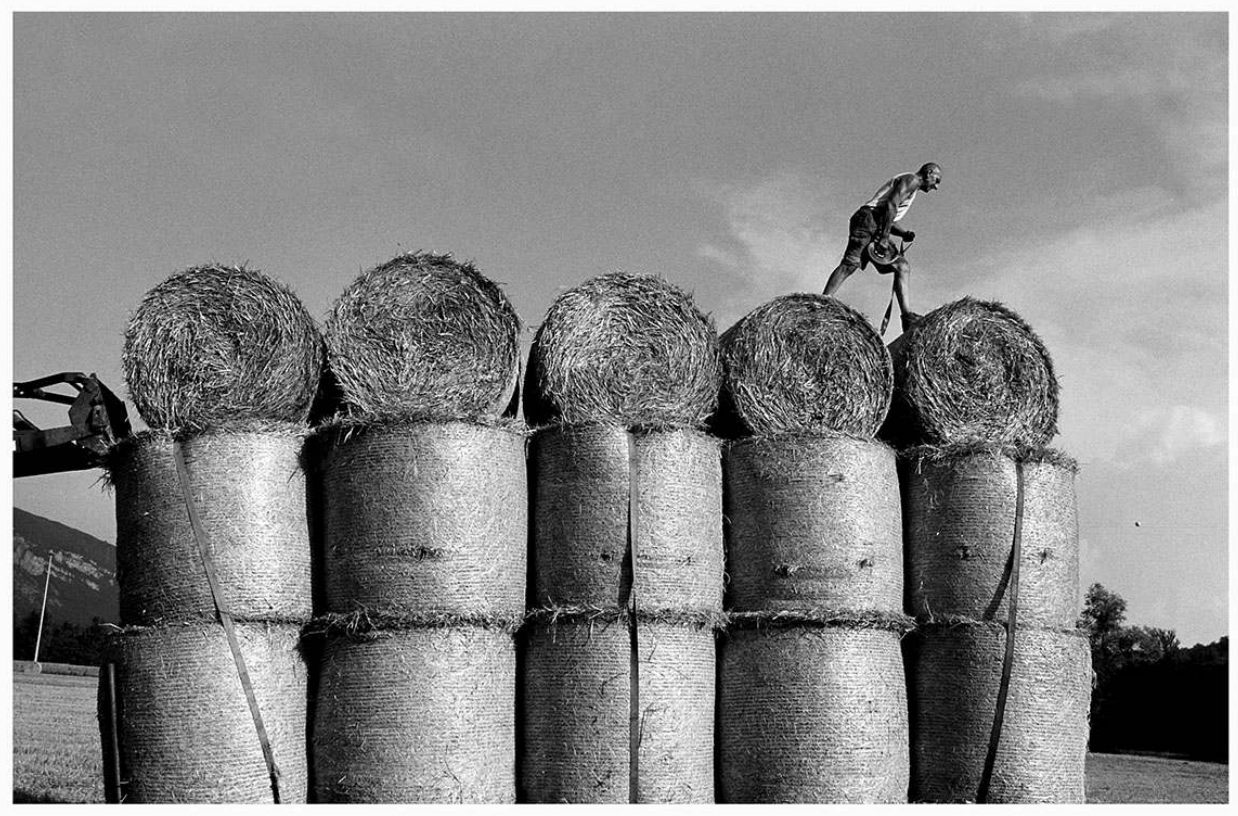

Portrait de Guy Trichet dans son exploitation. Coll. départementale des Musées de l'Ain, $n^{\circ}$ inv. 2006.100.25.

Phot. A. Passerat 2006. Conseil départemental de l'Ain, direction des Musées départementaux.

12 - musée du Revermont (Cuisiat), avec la conversion du territoire de la viticulture à l'élevage laitier, à la suite de la crise du phylloxéra, et surtout son potager-verger conservatoire d'espèces et variétés anciennes, locales et rares ;

- musée de la Bresse (Saint-Cyr-sur-Menthon), avec le domaine agricole, la volaille ${ }^{19}$, le lait, le beurre et la crème, et de façon plus générale, une approche anthropologique de l'alimentation et de la relation entre l'homme et son environnement, dans un parti pris de dialogue entre les disciplines et les arts ;

14 - musée de la Dombes (Villars-les-Dombes), avec la pêche et la chasse ;

15 - musée du Pays de Gex (Fort l'Ecluse, Léaz) avec les questions des circulations, de la frontière et de la contrebande.

16 Au cours des années 2000, le programme initial évolue. L'acquisition de l'important fonds des Soieries Bonnet en 2002, à la suite de la fermeture de l'usine de Jujurieux, et le transfert du musée d'Histoire de la Résistance et de la Déportation de l'Ain et du Haut-Jura en 2003 apportent une nouvelle coloration et font entrer dans les collections départementales des objets porteurs d'un éclairage complémentaire sur les questions alimentaires : vie quotidienne sous l'Occupation (tickets de rationnement, cartes de jardinage, cuisine des restrictions), matériel des maquis, menus de camps de prisonniers, d'une part; fonds relatif à l'école ménagère du pensionnat, à l'alimentation ouvrière et patronale, de l'autre ${ }^{20}$ (fig. 9). 
Figure 9

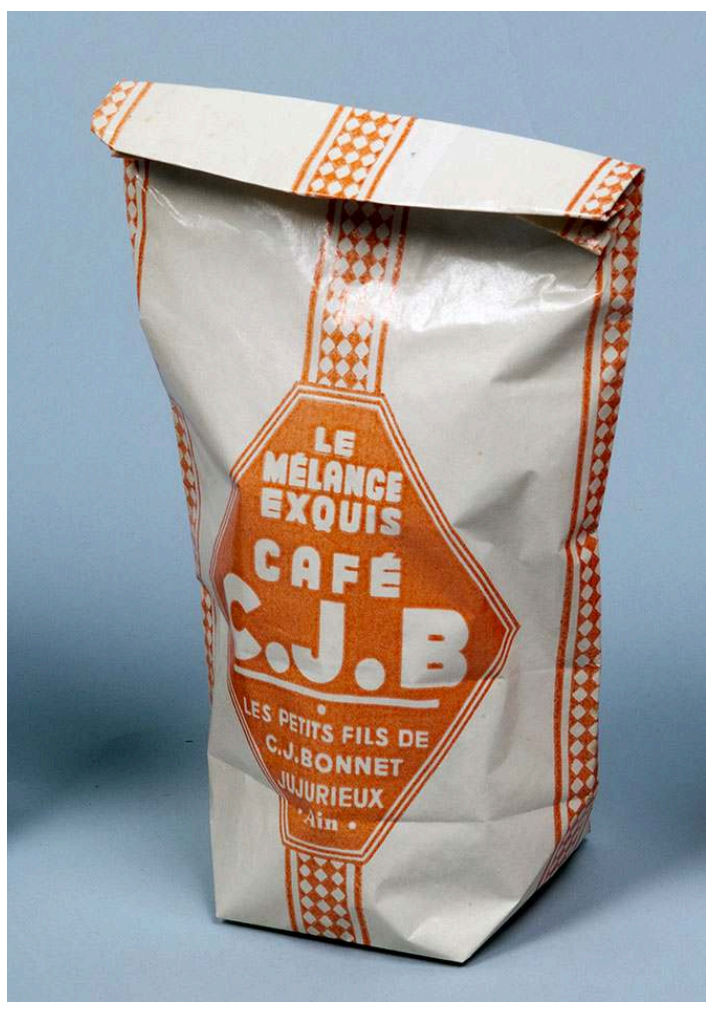

Paquet de café du magasin d'alimentation de l'usine C.-J. Bonnet à Jujurieux, fermé en 1963. Coll. départementale des Musées de l'Ain, n J2002.06.1295.

Phot. Jorge Alves. (C) Conseil départemental de l'Ain, direction des Musées départementaux de l'Ain.

La constitution de cette collection s'est également accompagnée de collectes de témoignages et de campagnes photographiques permettant de documenter les objets par la préservation d'un patrimoine culturel immatériel qui y est rattaché.

\section{Un laboratoire d'approches croisées représentatives de la diversité culturelle du territoire}

Ainsi, sans développer de manière formelle une approche globale de la thématique, le patrimoine gastronomique départemental s'est construit dans les collections muséales par juxtaposition de petites touches et de grands aplats, prenant forme au fil des projets patrimoniaux et culturels de la direction des Musées départementaux. Il n'a pas forcément toujours été exprimé dans les termes mêmes de " gastronomie ", mais plutôt en termes d'anthropologie de l'alimentation, de production agricole, d'ethnobotanique et de conservation des espèces végétales, de vie quotidienne, et aussi d'excellence et d'exception (produits, chefs...). Dès les années 1990, l'approche du musée de la Bresse passe notamment par la constitution d'une importante collection de menus : menus de fêtes des habitants de l'Ain, menus de restaurants, de croisières, menus politiques et présidentiels permettant d'illustrer les usages gastronomiques des produits régionaux. À partir des années 2000, l'approche du musée de la Bresse se renforce autour des figures des grands chefs et restaurants (cartes et menus, vaisselle) (fig. 10) et des arts de la table. 
Figure 10

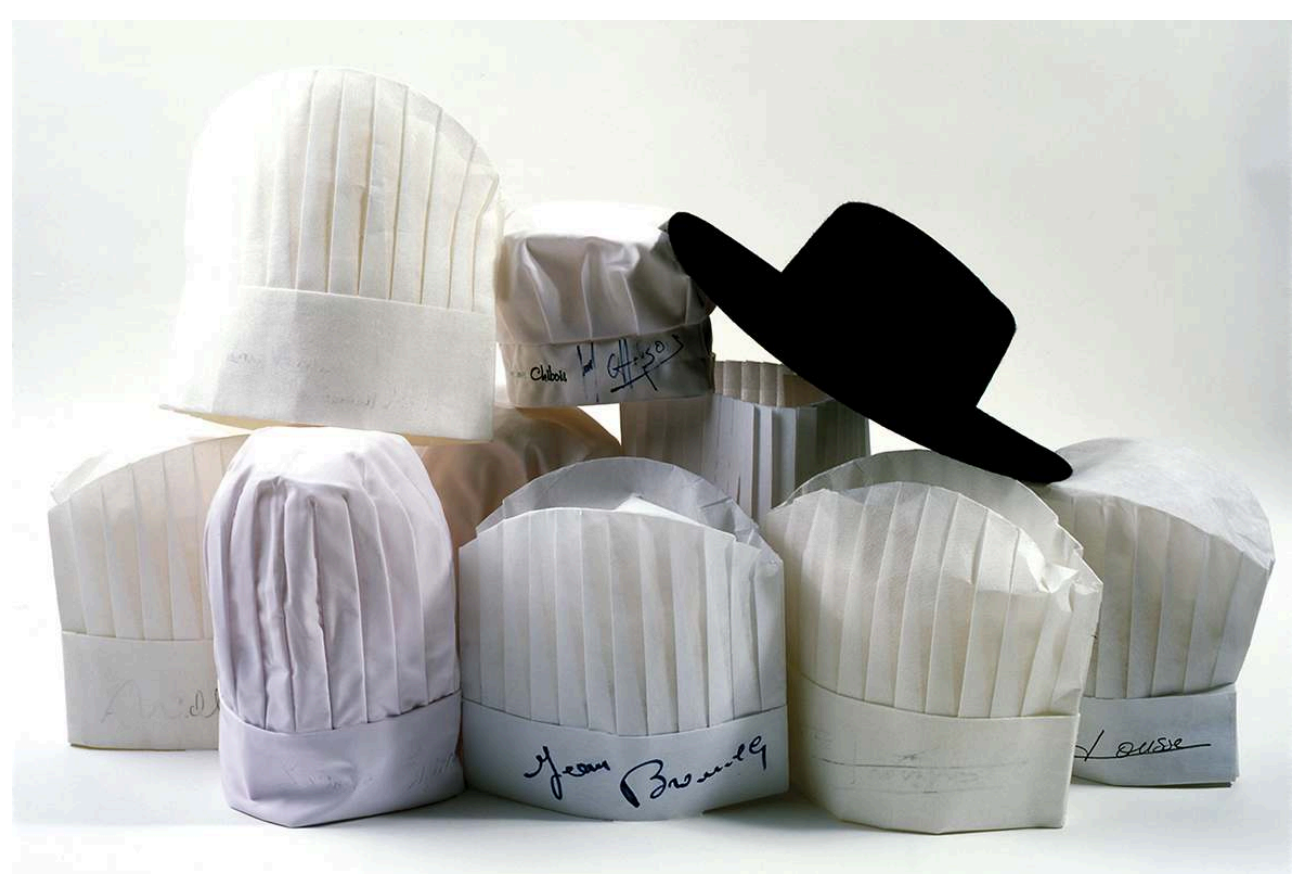

Toques dédicacées de grands chefs. Les collections du musée de la Bresse-Domaine des Planons comptent 95 toques dédicacées, parmi lesquelles celle de Paul Bocuse, Georges Blanc, Bernard Loiseau, Pierre Troisgros, Anne-Sophie Pic, Marc Veyrat, Jean Brouilly et Philippe Jousse, collectées à l'occasion de l'exposition «Toqués de cuisine » présentée en 2006 et 2007, commissariat Agnès Bruno et Céline Chanas.

Phot. Georges Rivalain, 2006. (C) Conseil départemental de l'Ain, direction des musées départementaux de l'Ain.

Au-delà de la constitution des collections, les actions menées par les musées ont permis de valoriser le patrimoine alimentaire de l'Ain, selon des approches propres aux problématiques développées sur les différents musées du territoire : une approche liant produits et territoire au musée de la Bresse ${ }^{21}$; une démarche plus ancrée sur les personnes, producteurs ou "gastronomades», au musée du Bugey-Valromey ${ }^{22}$. Au musée du Revermont, la singularité de la collection vivante a fourni le support ou le cadre pour des actions innovantes et des projets au croisement des arts, de l'agriculture et de la restauration gastronomique : mise en place d'un réseau de "conservateurs volontaires ", chargés de la multiplication des semences et de la description des variétés conservées au musée (fig. 11), collaborations entre chefs et photographes autours des collections végétales ${ }^{23}$. L'exposition «La Racine des légumes » présentait en 2013 le travail de la photographe Jacqueline Salmon, en collaboration avec le maraîcher Gilles Béréziat ${ }^{24}$, autour de portraits de légumes, vus comme jamais, c'est-à-dire dans leur intégralité, des feuilles aux racines et tout juste sortis de terre ${ }^{25}$. La notion de biodiversité cultivée et de savoirs ethnologiques liés aux plantes est sous-jacente dans l'ensemble des actions ${ }^{26}$. Au musée de la Résistance et de la Déportation de Nantua, l'exposition «Les Jours sans ${ }^{27}$ évoquait en 2018 les difficultés d'approvisionnement de la population civile et des maquis. 
Figure 11

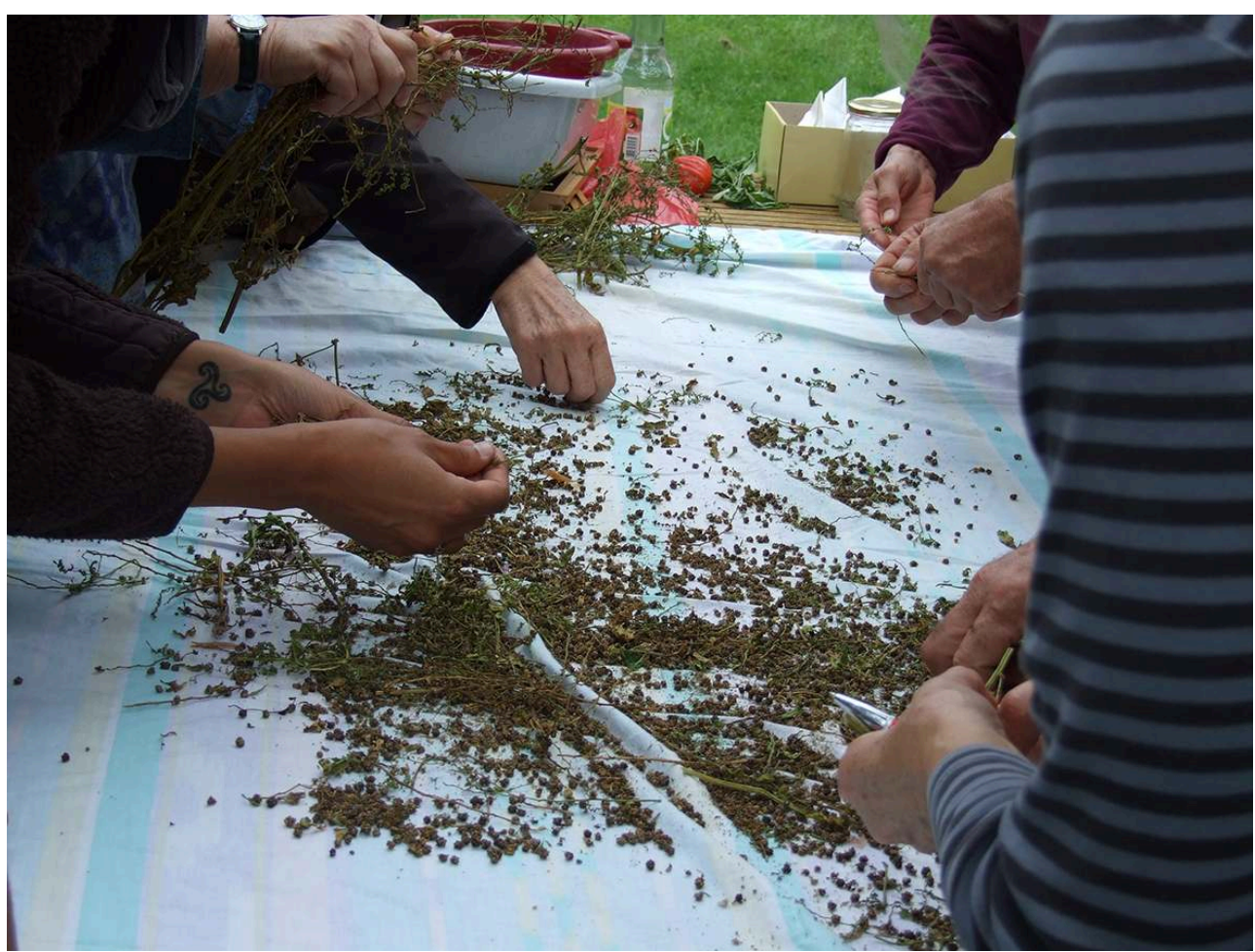

Récolte de semences à l'occasion d'une formation « graines » au musée du Revermont, septembre 2014. L'animation du réseau de conservateurs volontaires et les actions de formation sont menées en partenariat entre le musée et l'Association des amis de Treffort-Cuisiat et du musée du Revermont- réseau Solimence.

(c) Solimence.

L'ensemble de ces fils tissés de façon empirique prend un sens particulier à la lecture rétrospective et vient dessiner le canevas d'une vraie richesse d'approche et de ressources sur les questions liées au patrimoine gastronomique. La diversité géographique et culturelle du territoire départemental fait de l'Ain un laboratoire particulièrement intéressant pour illustrer le lien entre sociétés, économies et culture alimentaire. Ainsi, l'ethnologue Nicolas Millet distingue «deux modes de cuisson qui distribuent les recettes de part et d'autre de la rivière Ain : le mijoté (rive droite) et la fricassée (rive gauche) $\aleph^{28}$. Il explique cette différence par les caractéristiques historiques des terroirs et des parcelles cultivées favorisant, en Bresse, « une cuisine roborative et en quantité suffisante pour nourrir l'ensemble des actifs sur l'exploitation, [...] une cuisine qui puisse "attendre" le retour des équipes", et au contraire, en Bugey, une "cuisine de prises ", associant chasse et ramassage, saisie "sur le vif », répondant au "rythme du polyculteur appelé à d'autres tâches qui ne peuvent attendre ».

\section{Le patrimoine gastronomique, un objet insaisissable?}

\section{Un concept antinomique?}

21 Par nature, le patrimoine gastronomique est difficile à appréhender. Doit-on s'attacher aux produits, aux pratiques, aux savoir-faire, aux représentations? Comment 
patrimonialiser le goût? Quelles sont les traces matérielles de la gastronomie? Le périmètre de la patrimonialisation n'est-il pas condamné à se situer « autour » de son objet, dont le cœur, fugace, ne réside que dans l'instant d'une rencontre éphémère entre un patrimoine périssable et un public qui l'est de même?

Dans son acception large, celle «inclusive» de Brillat-Savarin, le patrimoine gastronomique relève à la fois du quotidien et de l'exceptionnalité, de la banalité de l'instant présent et d'une "mission impossible» en termes de conservation (conservation des biens alimentaires, évolution du vivant). Il renvoie à des questions intimes, identitaires, comme à des logiques d'ouverture, de circulations et d'échanges : au contraire d'un patrimoine figé, il est représentatif de dynamiques qui seules le font perdurer par la transmission.

Au-delà des besoins primaires de l'alimentation, la gastronomie est le "propre de l'homme ", probablement identifiable au "supplément d'âme " qui rattache un être à l'humanité, même dans les conditions les plus effroyables. Parmi les collections départementales se trouvent ainsi plusieurs carnets, rédigés dans les camps de concentration entre le printemps 1944 et l'année 1945, par trois femmes déportées: Germaine Coupat, Marie Planchon et Lucienne Volland. Sur des supports de papier récupérés, découpés et reliés avec des ficelles de fortune issues des paillasses, des centaines de recettes de cuisine sont consignées, comme un moyen de garder un lien avec la vie et l'humanitée ${ }^{29}$ (fig. 12).

\section{Figure 12}

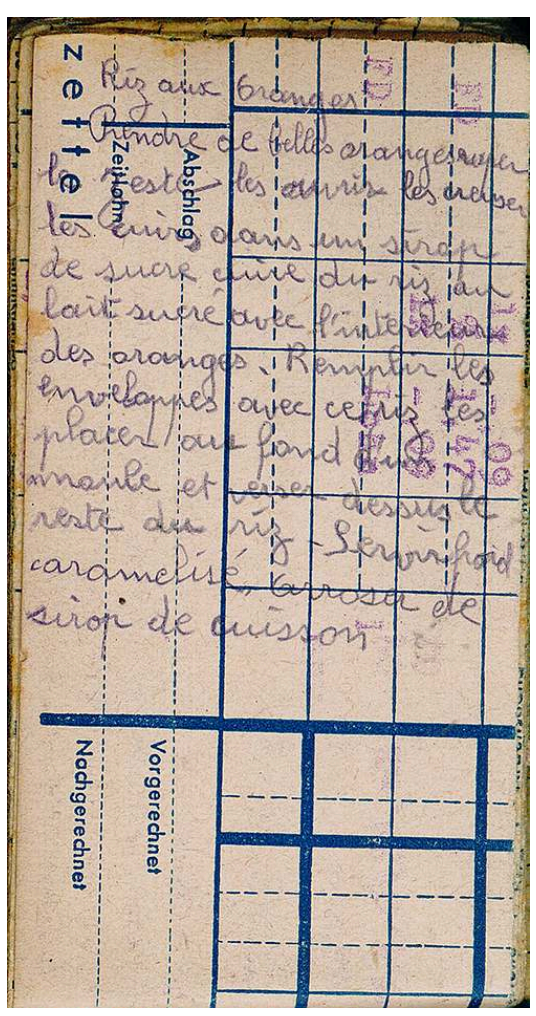

Page de l'un des carnets de recettes de Lucienne Volland, écrit durant sa déportation en camp de concentration. Coll. départementale des Musées de l'Ain, n inv. N2000.4.1.

(c) Conseil départemental de l'Ain, direction des Musées départementaux de l'Ain. 


\section{Une thématique en évolution constante, aux prises avec les enjeux les plus contemporains}

d'interconnexion de points de vue et de disciplines, apparait d'autant plus quand il s'agit de saisir l'insaisissable. La difficulté à appréhender la notion de patrimoine gastronomique et ses formes de patrimonialisation débouche ainsi sur une certaine plasticité, le patrimoine gastronomique évoluant avec la notion de patrimoine ellemême et la façon dont les musées de société, notamment, s'en saisissent.

De marqueur pour la construction identitaire d'un territoire, de composante d'une culture matérielle incarnée par les arts et traditions populaires, on observe que le champ du patrimoine gastronomique s'est diversifié et élargi à mesure que la notion de patrimoine s'enrichissait et que des enjeux de société se précisaient. L'inscription du repas gastronomique des Français sur la Liste représentative du patrimoine culturel immatériel de l'humanité (Unesco) en 2010 en est une illustration. Au musée de la Bresse notamment, les années 2010 sont ainsi l'occasion d'explorer la thématique de l'alimentation sous d'autres facettes : patrimoine culturel immatériel ${ }^{30}$, approche par l'art contemporain ${ }^{31}$, le genre (fig. 13) et la diversité culturelle ${ }^{32}$.

Figure 13

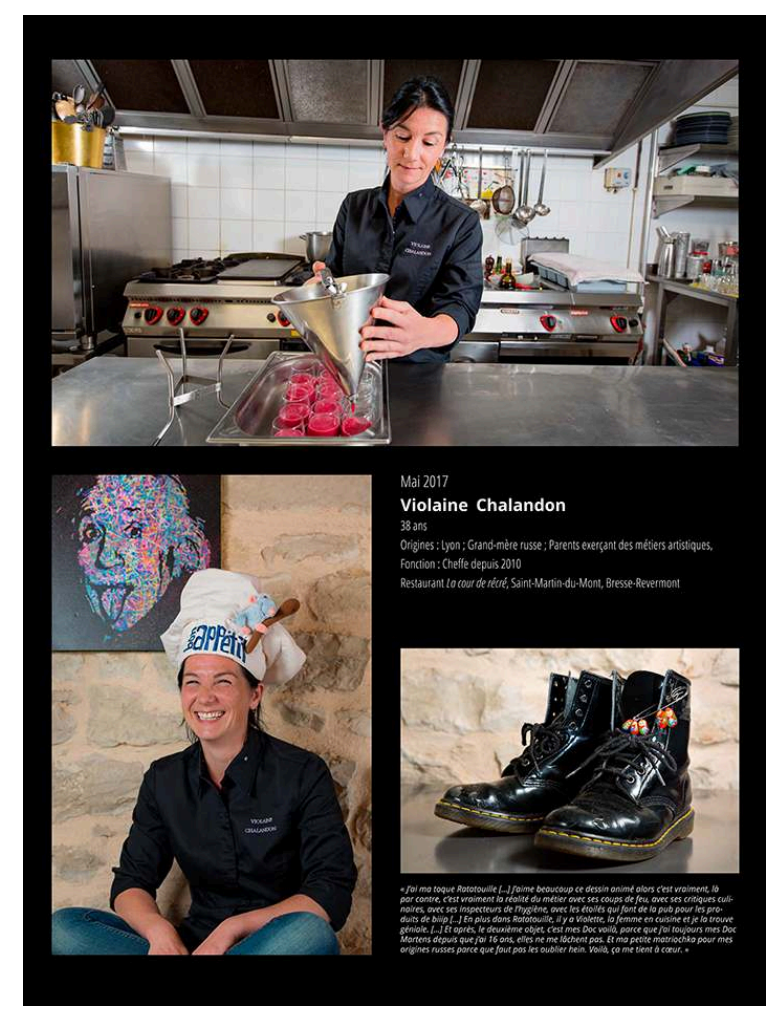

Panneau extrait de l'exposition capsule «Éleveuses de volailles de Bresse/Cheffes de restaurant. Regards sur les femmes de l'Ain », présentée au musée de la Bresse à l'occasion des journées du Matrimoine en Auvergne-Rhône-Alpes (2018), commissariat Aurélie Faivre.

(C) Michel Armenta

Quelle que soit l'approche, la gastronomie entendue au sens large, en tant que «fait social total », parle au visiteur et l'engage dans une relation personnelle au patrimoine. 
Elle constitue donc un lieu de rencontre particulièrement fécond entre le patrimoine et les publics. Les enjeux de légitimité démocratique des institutions culturelles et de renouvellement du lien avec les publics peuvent donc utilement être explorés à partir de cette thématique : mobilisation des habitants et des acteurs dans la co-construction des expositions ${ }^{33}$, création d'installations artistiques (fig. 14), dimension festive ${ }^{34}$, expérimentation, dégustations et formations...

Figure 14

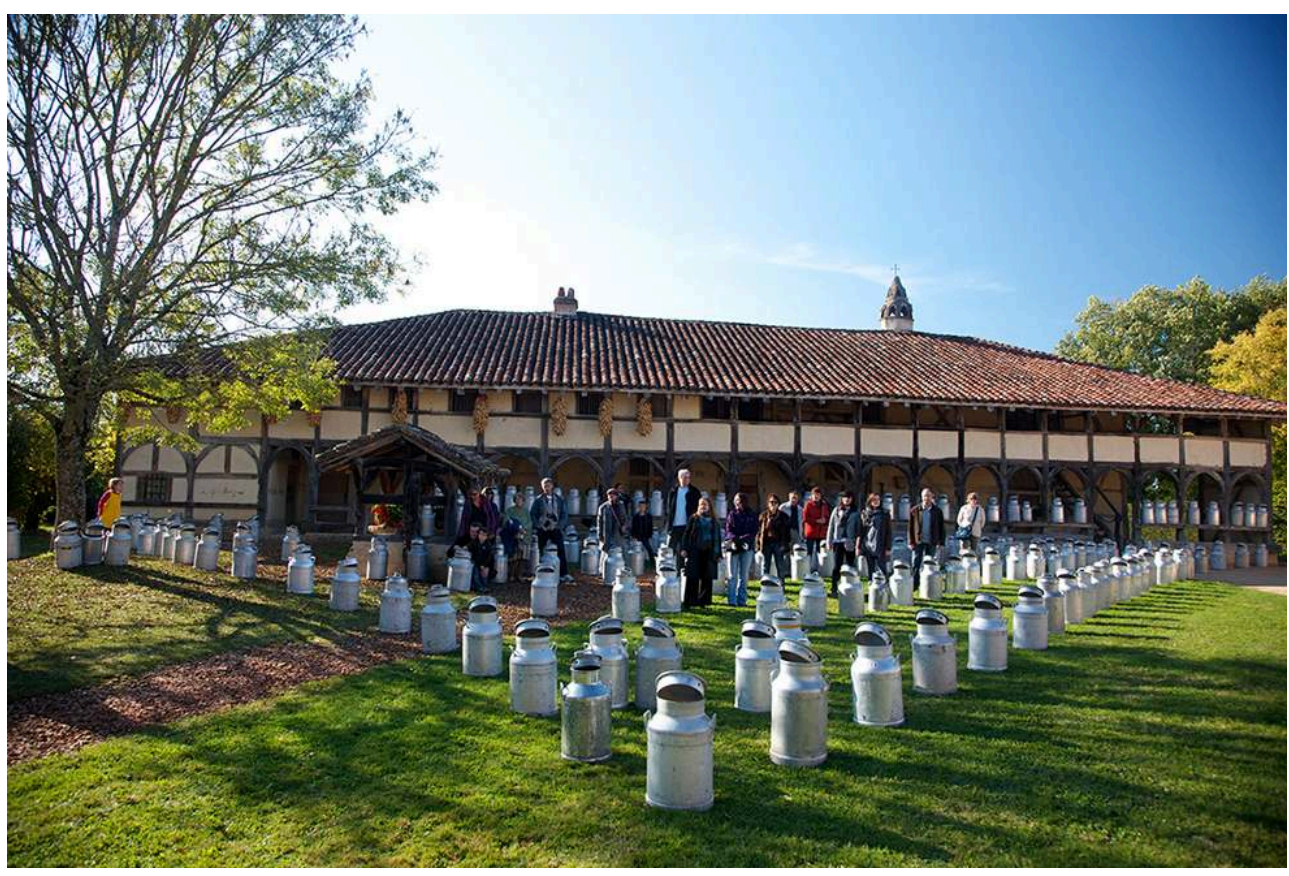

Une des installations événementielles réalisées avec le public par le photographe Gérard Benoît à la Guillaume lors du Week-end du goût 2011, dans le cadre de l'exposition photographique « Bidons sans frontières ».

(c) G. Benoît à la Guillaume/Conseil départemental de l'Ain, direction des Musées départementaux, 2011.

27 Aujourd'hui, la notion de patrimoine gastronomique est réinterrogée par de forts enjeux sociétaux, au premier rang desquels les enjeux climatiques et environnementaux, qui ont conduit au lancement en 2019 du Livre blanc de la gastronomie responsable ${ }^{35}$. Une nouvelle gastronomie émerge, «tournée vers la valorisation des terroirs, la protection de la planète, la santé des clients, mais aussi le bien-être des hommes et des femmes qui font ce métier $»^{36}$. Le patrimoine gastronomique peut devenir une ressource pour le développement économique et sociétal futur, notamment en termes de biodiversité domestique, de savoir-faire écologique et de véhicule des valeurs d'échange, de partage et d'humanité. 


\section{NOTES}

1. - ROUFF, Marcel, et SAILLAND, Maurice Edmond, dit CURNONSKY. La France gastronomique. Guide des merveilles culinaires et des bonnes auberges françaises. Paris : F. Rouff, 1921-1926, La Bresse, le Bugey, le pays de Gex, 1921.

2. - La figure 1 représente le critique gastronomique à l'immense renommée, Curnonsky qui a fait l'objet de nombreuses célébrations, de son vivant et à titre posthume. Ce menu-hommage qui le représente en plein toast au milieu d'un tourbillon de créations culinaires, signé Georges Villa pour l'iconographie, a été donné le 28 octobre 1952 par le chef René Viaux à l'occasion des 80 ans du «prince élu des gastronomes ", dans le cadre d'un dîner de gala organisé par l'Académie des gastronomes, l'Académie des gais gentilshommes gastronomes, l'Académie des psychologues du goût, l'Administration mutuelle, les Amis de la table, l'Association des gastronomes régionalistes, l'Association de la presse gastronomique et des écrivains gastronomes, le Cercle des gourmettes, la Chaîne des rôtisseurs, le Club des Cent, le Club gastronomique Prosper Montagné, le Comité du tourisme gastronomique du TCF, les Compagnons de la Belle Table, la Confrérie des chevaliers de la Poularde, les Purs-Cent, la section d'Arts gastronomiques du Salon des arts ménagers et les Disciples de Brillat-Savarin.

3. - SAILLAND, Maurice Edmond, dit CURNONSKY. "Vue cavalière de la Principauté des Gastronomes ». Marianne, 15 août 1934, p. 1.

4. - GOSSELIN, Théodore, dit G. LENOTRE. «Au pays de Brillat-Savarin ». Le Temps, 25 mai 1922, p. 3. L'auteur emploie également les expressions de "terre promise", de "paradis des nobles lippées » et ajoute, au sujet de Belley (Bugey) « en cette région mille fois bénie, tout le monde naît gourmand - comme on naît colonel au Guatemala ».

5. - MONMARCHÉ, Georges. Franche-Comté, Monts Jura [1946]. Paris : Hachette, coll. «Guides bleus ", 1961, p. lviii.

6. - BRILlAT-SAVARIN, Jean-Anthelme. Physiologie du goût [1828]. Paris: G. de Gonet, 1848, p. 27-28.

7. - DROUARD, Alain. Les Français et la table. Alimentation, cuisine, gastronomie du Moyen Âge à nos jours. Paris : Ellipses, 2005.

8. - Voir le portrait de la mère Prusse sur le site: http://www.archives.ain.fr/ark:/22231/ vtaad908600c950e88c [consulté le 23/10/2019].

9. - $A B C$, journal d'information illustré, Genève, 20-21 avril 1912, cité dans l'exposition "L'Ain au menu », musée du Bugey-Valromey à Lochieu, 2018-2019 (commissariat Aurélie Faivre et Suzanne Lantelme, sous la direction de Delphine Cano).

10. - PERNOLLET, Jean-Luc. "L'hôtel-restaurant Pernollet : 163 ans d'histoire », texte extrait de l'exposition «L'Ain au menu », musée du Bugey-Valromey.

11. - CSERGO, Julia. « L'emblème du terroir ». Dans MEILLER, Daniel (dir.). Le Mangeur du $21^{e}$ siècle/ Les aliments, le goût, la cuisine et la table. Dijon : Éducagri, 2003, p. 147-158.

12. - CANO, Delphine (dir.). Terroir et étiquettes, une histoire d'image(s). Bourg-en-Bresse : conseil départemental de l'Ain, 2010, 24 p. Livret de l'exposition éponyme présentée au musée de la Bresse-Domaine des Planons en 2010 et 2011 (commissariat Delphine Cano, Céline Chanas et Pascale Court).

13. - La construction de cette image passe aussi par les Expositions universelles. Ainsi, pour la première fois en 1867, un costume de Bressane, confectionné par la maison Babin à Paris, est présenté au Champ-de-Mars, parmi les 600 «spécimens des costumes populaires de diverses contrées" (voir CANO, Delphine (dir.). Émaux de Bresse, joyaux du quotidien. Bourg-en-Bresse : conseil général de l'Ain, 2014, p. 130). 
14. - BÉRARD, Laurence, MARCHENAY, Philippe. «Les gaudes en Bresse, pratiques techniques et consommation ». Dans FRANCONIE, Hélène, CHASTANET, Monique, SIGAUT, François (dir.). Couscous, boulgour et polenta: transformer et consommer les céréales dans le monde. Paris: Karthala, 2010 ; Iid. "Un exemple de "non patrimonialisation": la carpe en Dombes». Colloque international «Les Systèmes agroalimentaires localisés: produits, entreprises et dynamiques locales ", Montpellier, 16-18 oct. 2002, communication non publiée.

15. - Citons, à titre d'exemple, les lancements des marques « Poissons de Dombes » puis «Cuir de carpe de Dombes» en 2012 et 2015 par l'Association de promotion du poisson des étangs de la Dombes (APPED), ou encore l'inscription, en 2017, de la pêche aux poissons d'eau douce des étangs de la Dombes à l'Inventaire national du patrimoine culturel immatériel (réf. 2017_67717_INV_PCI_FRANCE_00380), voir le site: http://www.culture.gouv.fr/Thematiques/ Patrimoine-culturel-immateriel/L-inventaire-national/Inventaire-national/Fiches-de-l-

Inventaire-national-du-PCI/Savoir-faire-de-l-artisanat-traditionnel). Quant aux gaudes, la biscuiterie Gaudélices, créée en 2011 à Tossiat, développe les sablés à la farine de gaudes parmi toute une déclinaison de biscuits conçus avec cette même farine de maïs torréfiée, consommée historiquement en bouillie ou poêlée une fois coupée en tranches.

16. - Ainsi, la renommée de la volaille de Bresse a bénéficié notamment du soutien du comice agricole, qui organise la première Glorieuse, concours de volailles fines, à Bourg-en-Bresse, en 1862, du Comité interprofessionnel de la volaille de Bresse après l'obtention de l'AOC en 1957, de la Confrérie des poulardiers de Bresse, créée en 1962, des nombreux gastronomes (dont BrillatSavarin) et de chefs (tels Paul Bocuse ou Georges Blanc) qui s'en sont faits les ambassadeurs. Parmi les autres confréries gourmandes de l'Ain visant la promotion de produits locaux, on peut citer aussi l'Académie Granet (1945), l'Ordre des commandeurs du Bugey (1982). Pour aller plus loin, voir le fonds sonore «Les confréries alimentaires de l'Ain» sur le site: https:// cmtra.hypotheses.org/2482 [consulté le 25/10/2019].

17. - Arrêté du 25 juin 1938. Voir dans la base Mérimée : notice PA00116544.

18. - La Figure 8 présente le portrait de Guy Trichet dans son exploitation. Agriculteur de Souclin (Ain), il combine des activités d'élevage bovin, de production céréalière, d'entreprise forestière et de service public de déneigement. Cette photographie a été présentée, parmi d'autres portraits, dans l'exposition « Les Médiateurs de la terre, regards sur la polyactivité de moyenne montagne en Rhône-Alpes » au musée du Bugey-Valromey en 2007 et 2008, commissariat Agnès Bruno et Suzanne Lantelme. L'exposition restituait l'enquête ethnographique menée par Bénédicte Bonnefoy-Salavert pour la conservation départementale des Musées des pays de l'Ain, avec le soutien de la DRAC Rhône-Alpes et de l'ISARA.

19. - Présente physiquement grâce à l'élevage de Cyril Degluaire dans les espaces extérieurs du domaine.

20. - La figure 9 présente un paquet de café du magasin d'alimentation de l'usine C.-J. Bonnet à Jujurieux, fermé en 1963. Véritable ville dans la ville, la manufacture des Soieries Bonnet réunissait, en plus de son outil productif, une exploitation agricole et viticole, un four à pain, un terminus de tramway et une série d'infrastructures sociales: pensionnat et cités ouvrières, crèche-garderie, infirmerie, école ménagère, magasin d'habillement et d'alimentation... Les habitants de Jujurieux se souviennent de l'odeur du café grillé, torréfié sur place.

21. - Expositions « Terroir et étiquettes, une histoire d'image(s) », et «L'Ain au menu, un art de la gastronomie » (2018-2019, musées de la Bresse et du Bugey-Valromey).

22. - Expositions «Les Médiateurs de la terre» (2007) (commissariat Agnès Bruno, Suzanne Lantelme et Frédéric Bravard); "Gertrude Stein et le Bugey»(2012-2013) (commissariat Delphine Cano, Benjamin Gurcel et Suzanne Lantelme) et «L'Ain au menu, un art de la gastronomie ». 
23. - En 2014, l'exposition «Transparences culinaires » associait ainsi le chef Didier Goiffon, du restaurant La Marelle, à Péronnas (Ain), et le photographe Pierre-François Couderc, autour d'une exploration de la matière des légumes.

24. - Maraîcher à la ferme du Bioux, proche de Bourg-en Bresse.

25. - Au sujet de ce travail photographique, Jean-Christophe Bailly notait: «Si l'on devait en référer à la question du genre, on verrait que ces images, par le statut qu'elles accordent aux légumes ainsi représentés, ne peuvent être apparentées au régime de la nature morte, dans lequel tout repose d'abord sur l'arrangement et la composition. Et c'est avec surprise qu'on les verrait plutôt glisser vers une action qui serait davantage celle qu'exerce le portrait, voire le nu. Le nu, parce que soustraits à l'habituel habillage jardinier, marchand ou culinaire, ces légumes sont pour ainsi dire autorisés à se montrer sans nul appareil. Et du portrait parce qu'il ne s'agit à chaque fois que d'un individu singulier [...]. À une époque où l'on parle tellement de biodiversité, ces images - modestes et orgueilleuses à la fois - viennent utilement rappeler que le divers n'est ni un label ni un argument mais, en vérité, une condition d'existence » (BAILLY, Jean-Christophe. La Racine des légumes. Bourg-en-Bresse : conseil général de l'Ain, 2013, p. 6-7).

26. - Parmi les expositions, on peut citer «Oser la biodiversité. Le patrimoine agricole du futur ?»(2016-2017), commissariat Delphine Cano et Agnès Ducaroy.

27. - Exposition réalisée par le Centre d'histoire de la Résistance et de la Déportation de Lyon, complétée par des collections et des témoignages en lien avec le département de l'Ain (commissariat Florence Saint Cyr-Gherardi pour la partie locale). Au sujet du ravitaillement des maquis, citons le témoignage étonnant du capitaine Owen Denis Johnson, agent du Special Operations Executive arrivé en France en octobre 1943: "Il faut dire que la "Montagne" a survécu grâce à la générosité de la "Plaine". De la corne d'abondance "Bresse-Dombes" sortaient tous les produits de la ferme, transportés par des camions gazogènes pleins à craquer, au travers du grillage allemand et à la barbe des réquisitions du gouvernement de Vichy. [...] La "Montagne" produisit quand même un produit qui était le sien : le comté. [...] Comme le pain à l'époque était rare, on confectionnait des sandwichs de la manière suivante : deux tranches de comté avec une tranche de pain au milieu " (anecdotes dactylographiées par O. D. Johnson en février 1987, cité dans VEYRET, Patrick. Une porte ouverte sur le maquis. Châtillon-sur-Chalaronne : La Taillanderie, 2002, p. 118).

28. - MILLET, Nicolas. «Le mijoté et la fricassée. De quelques pratiques culinaires en Bresse et Bugey (Ain) ». Texte extrait de l'exposition «L'Ain au menu, un art de la gastronomie ».

29. - La figure 12 représente une page de l'un des carnets de recettes de Lucienne Volland, écrit durant sa déportation en camp de concentration. Après avoir apporté son aide aux réfractaires au STO cachés dans la grotte de l'Ours près de Chougeat, Lucienne Volland est arrêtée à Matafelon (Ain) lors de l'opération de répression Frühling en avril 1944. Déportée au camp de concentration de Ravensbrück, elle est ensuite transférée au kommando de Zwodau sous le matricule 51536.

30. - Exposition «Le Repas gastronomique des Français en Bresse, un art de vivre »(2013), commissariat Aurélie Faivre.

31. - Installations Akousmaflore et Le Cabinet de curiosité contemporain par Scénocosme, pour le $2^{\mathrm{e}}$ Rendez-vous de l'art contemporain au musée de la Bresse (2013).

32. - Exposition "Mémoire de Bressans" (2018) sur des Bressans d'origine étrangère, en partenariat avec les associations burgiennes Ain'Pacte et Alfa 3A, commissariat d'Aurélie Faivre.

33. - Comme cela a pu être expérimenté dans la déclinaison bugiste de «L'Ain au menu, un art de la gastronomie ».

34. - Concours des chefs et festin des Planons en 2017.

35. - Voir sur le site: https://alimentation-generale.fr/wp-content/uploads/2018/10/LivreBlanc-de-la-Gastronomie-Responsable_Les-bonnes-feuilles_Good-France-2019.pdf [consulté le 25/10/2019]. 
36. - Interview de René Redzepi, chef du restaurant Noma à Copenhague, reconnu «meilleure table du monde » à quatre reprises dans les années 2010 par le jury du San Pellegrino World's 50 Best Restaurants (entretien par F.R. Gaudry, le 7 avril 2018, voir le site: https://www.lexpress.fr/ styles/saveurs/restaurant/noma-hors-norme_1997565.html [consulté le 25/10/2019]).

\section{RÉSUMÉS}

L'Ain est une terre de gastronomie, qui a vu naître Brillat-Savarin et est décrite par les gastronomes comme celle de la « perfection » culinaire. Espace d'échanges et de circulation entre vallée du Rhône, la Suisse et la Bourgogne, terre d'approvisionnement pour les villes voisines, la Bresse a construit son identité régionale en particulier sur la renommée de ses produits du terroir, et notamment de la volaille de Bresse. Aujourd'hui, le positionnement majeur du musée de la Bresse, à Saint-Cyr-sur-Menthon (Ain), autour du patrimoine gastronomique de l'Ain, s'inscrit dans une volonté de développement touristique du territoire du département mais aussi dans la suite logique d'une politique de collecte, de conservation et de valorisation du patrimoine local et national de l'alimentation menée par la direction des Musées départementaux de l'Ain depuis trente-cinq ans. Une lecture rétrospective de ces actions dessine un canevas riche des échanges entre approches et disciplines, qui reflète l'histoire muséale singulière du département: collections végétales de variétés locales et rares dans le potager-verger conservatoire du musée du Revermont, domaine agricole, menus et grands chefs au musée de la Bresse, cueillette et cuisine dans l'esprit de Brillat-Savarin et de Gertrude Stein en pays bugiste, pêche et chasse en Dombes, contrebande en pays de Gex, patrimonialisation des pratiques alimentaires en temps de guerre au musée de la Résistance et de la Déportation à Nantua, ou encore patrimoine de l'alimentation ouvrière aux Soieries Bonnet, à Jujurieux. Le croisement de ces approches permet d'aborder la notion de patrimoine gastronomique dans sa complexité et de suivre son évolution au fil de l'enrichissement du champ patrimonial et des mutations des musées de société. Aujourd'hui, le patrimoine gastronomique, patrimoine partagé, se trouve au cœur des enjeux sociétaux, tant climatiques que démocratiques.

The Ain department is well known as a land of gastronomy. The celebrated French author and gastronome Jean-Anthelme Brillat-Savarin was born there and gourmets call two of its regions, Bresse and Bugey, lands of culinary 'perfection'. The department is a place of exchanges and circulation between the Rhône Valley, Switzerland and Burgundy, largely dedicated to supplying food to neighbouring cities. The Bresse region, in particular, has a local identity built on the fame of its products, including its well-known Bresse poultry with its AOP label (protected designation of origin). Today, the Bresse Museum at Saint-Cyr-sur-Menthon is particularly keen to promote the gastronomic heritage as part of a general policy of encouraging tourism in the department. This is also a continuation of a policy of collecting, preserving and promoting the local and national gastronomic heritage initiated by the Ain museums service some 35 years ago. A retrospective analysis of these initiatives paints a rich picture of exchanges between different approaches and disciplines and underlining the specific history of the department's museums: collections of local and rare plant varieties in the garden of the Revermont Museum; agriculture, menus and figures of famous chefs at the Bresse Museum; food gathering and cooking in the spirit of Brillat-Savarin and the American author Gertrude Stein in Bugey; fishing and hunting in the Dombes; smuggling in Gex; wartime food practices at the Nantua Museum of Resistance and 
Deportation; the food of ordinary working men and women at the Bonnet silk factory in Jujurieux. The intersection of all these actions makes it possible to approach the notion of gastronomic heritage in all its aspects and all its complexity. We can follow its evolution through a period in which the whole field of heritage has seen considerable diversification and during which museums of society have also evolved. Today, the gastronomic heritage has become a shared heritage at the heart of various societal issues, including climate change and democracy.

\section{INDEX}

Keywords : Ain, Bresse, Bugey, Dombes, Genève, Lyon, Nantua, Revermont, Blanc (Georges), Bocuse (Paul), Brillat-Savarin, Curnonsky, Bonnet silk factory, food, biodiversity, concentration camps, chefs, ethnobotany, ethnology, 'gastronomades' festival, gastronomy, responsible gastronomy, gender, history, maquis, menus, museum, participation, heritage designation, polyculture, vegetable garden, tourism, Bresse poultry

Mots-clés : Ain, Bresse, Bugey, Dombes, Genève, Lyon, Nantua, Revermont, Blanc (Georges), Bocuse (Paul), Brillat-Savarin, Curnonsky, Soieries Bonnet, alimentation, biodiversité, camps de concentration, Chefs, ethnobotanique, ethnologie, gastronomades, gastronomie, gastronomie responsable, genre, histoire, maquis, menus, musée, participation, patrimonialisation, polyculteur, potager, tourisme, volaille de Bresse

\section{AUTEUR \\ DELPHINE CANO}

Conservatrice en chef du patrimoine, directrice des Musées départementaux, conseil départemental de l'Ain delphine.cano@ain.fr 